Scientific Journal of October 6 University

ISSN (Print): 2314-8640

ISSN (Electronic): 2356-8119

Published by October 6 University (C) All Rights Reserved

Available online at: http:// sjou.journals.ekb.eg
Citation: Elkattan et al., (2016). The Effect of Individual Blade Pitch Angle Control of Wind Turbine. Sci.J. of Oct. 6 Univ. 3(1), 66-74.

Copyright: (C) 2016 Elkattan et al., This is an open-access article distributed under the terms of the Creative Commons Attribution License, which permits unrestricted use, distribution, and reproduction in any medium, provided the original author and source are credited.

Review

\title{
The Effect of Individual Blade Pitch Angle Control of Wind Turbine
}

\author{
A. EIRahman M. Elkattan ${ }^{1, *}$, Aya Diab ${ }^{2}$, Zakaria Ghoneim ${ }^{2}$, M. Abdelhameed ${ }^{2}$ and Farid Tolba ${ }^{2}$ \\ ${ }^{1}$ Mechatronics Engineering Department, High Institute of Engineering - 6th October City, Cairo, Egypt \\ ${ }^{2}$ Mechatronics Engineering Department, Ain Shams University, Cairo, Egypt
}

Received: 8-11-2014/ Revised: 14-12-2014 / Accepted: 05-01-2015

\begin{abstract}
Because of this great interest of developing wind energy in the recent years which it's being one of the fastest growing energy resources. So it was necessary to seek to develop the control systems in wind turbine to reduce the loads on the turbine and on its blades specially and to improve the power that can we can take from the wind turbine.

This paper introduces a brief overview literature survey about "Individual Blade Pitch Control", this topic met with considerable interest in research at the last ten years.
\end{abstract}

Key Words: Individual Blade Pitch, aerodynamics loads, and wind turbine.

\section{Introduction}

In recent years, wind energy has become popular due to its inherent attribute of reproducible, resourceful and pollution-free characteristics against the rapid depletion and increasing environmental threats of conventional energy. Moreover, wind energy has been competing with conventional energy as a result of its cost reduction with technological advancements and incentives for adopting renewable energy since last decade. All these factors have caused wind power to become the fastest growing energy source.

The possibility of using individual pitch control for load alleviation has been suggested many times over the years, and more recently by Caselitz [1]. Recent work demonstrates that some very significant reductions in loading can be achieved and that the control algorithms required for this may be relatively simple. There are a number of reasons why the time may now be right to develop this idea commercially [2].

- As commercial turbines get larger, many of them now use individual pitch actuators anyway, since with careful design they can be considered as independent braking systems, obviating the need for a high-capacity shaft brake.

- The importance of load reduction becomes ever greater as turbines become larger and more flexible. Load reduction through 'intelligent' control systems becomes more attractive, compared with designing mechanical systems to cope with large loads, and processing power for control systems is no longer a limitation.

- The technique aims to reduce the asymmetric loads due to wind speed variations across the rotor disc, and these loads are becoming more significant as turbine rotors get larger with respect to the size of typical turbulent eddies in the wind.

- Through the use of the latest software tools, our understanding of the problem has increased and reliable methods for designing suitable control algorithms have been developed. The performance of these control algorithms can now be tested using very realistic simulations.

- The technique relies on sensors which can measure the asymmetric loads acting on the system, and load sensors with the necessary level of reliability are now becoming available. Various options for the positioning of load sensors are investigated in this article. It is also possible that other measurements could be used instead, such as accelerometers in each blade tip or lateral and vertical accelerometers in the nacelle.

In this situation, many small scale wind farms are connected to the distribution network while many wind farms of 50MW or more are directly connected to transmission network. These bring new challenges to the stability of power system. One of the challenges is that wind power is not constant and can fluctuate significantly below the rated power since wind power is proportional to the cube of the 
wind speed. The problems originated by the wind power fluctuations are as follows [3]:

- Wind power fluctuations may cause the grid frequency to fluctuate.

- Amount of absorbed reactive power by the induction generator from power grid is directly related to the active power generation. The variation in wind speed causes the fluctuation of the active power generation and thus the absorbed reactive power,

- Leading to voltage flicker at the buses of the power grid.

- Frequency fluctuation and voltage flicker provide poor power quality and originate instability problems in the power system, especially when there are loads sensitive to accept high voltage and frequency variations.

The purpose of the pitch angle control might be expressed as follows [4-7]:

1) Optimizing the power output of the wind turbine. Below rated wind speed, the pitch setting should be at its optimum value to give maximum power.

2) Preventing input mechanical power to exceed the design limits. Above rated wind speed, pitch angle control provides a very effective means of regulating the aerodynamic power and loads produced by the rotor.

3) Minimizing fatigue loads of the turbine mechanical component.

1 Wind turbine modeling

1.1 Power Capture of Wind Turbines

The power in the wind is proportional to the cube of the wind speed and may be expressed as $P=0.5 \rho A V_{W}^{\mathrm{a}}$

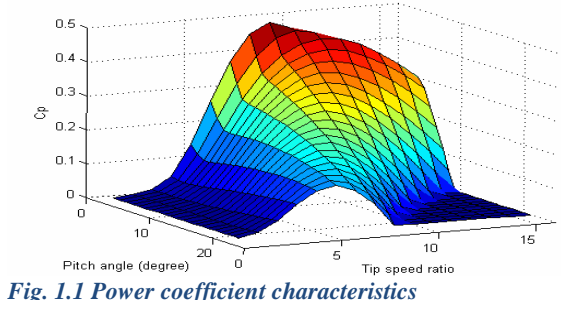

Where $\rho$ is air density, $\mathrm{A}$ is the area swept by blades

and is wind speed. A wind turbine can only extract part of the power from the wind, which is limited by the Betz limit (maximum59\%). This fraction is described by the power coefficient of the turbine, $\mathrm{Cp}$, which is a function of the blade pitch angle and the tip speed ratio. Therefore the mechanical power of the wind turbine extracted from the wind is

$$
\left.P_{W V}=0.5 C_{p}(\beta, \lambda) \rho A V_{W}^{a} \quad 1.2\right)
$$

Where $\mathrm{Cp}$ is the power coefficient of the wind turbine, $\beta$ is the blade pitch angle and $\lambda$ is the tip

speed ratio. The tip speed ratio is defined as the ratio between the blade tip speed and the wind speed $\lambda=\frac{\omega R}{W_{w}}$

Where $\omega$ the turbine rotor speed and $\mathrm{R}$ is is the

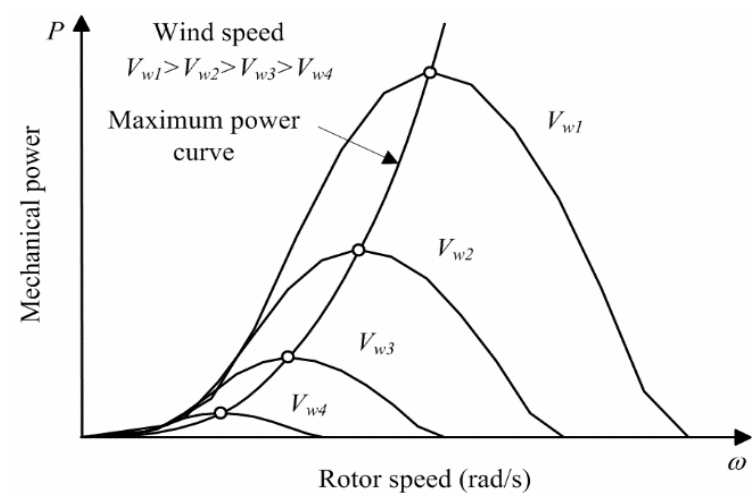

Fig.3.2.Mechanical power versus rotor speed

radius of the wind turbine blade $\mathrm{R}$.

Thus any change in the rotor speed or the wind speed induces change in the tip speed ratio leading to power coefficient variation. In this way, the generated power is affected. Fig. 3.1 shows a group of typical $\mathrm{Cp}-\lambda$ curves where optimum values of tip

speed ratio, $\lambda \mathrm{Opt}$, correspond to the maximum

power coefficient, Cp.max, Fig. 3.2 shows that the mechanical power converted from the turbine blade is a function of the rotational speed, and the

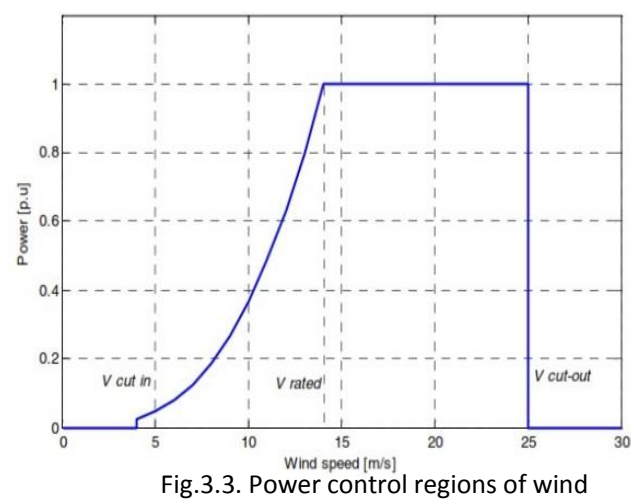

converted power is maximized at the particular rotational speed for various wind The typical power control regions of wind turbine are shown in Fig. 3.3. The turbine starts operating when the wind speed exceeds cut-in wind speed. The power captured by the turbine increases with the wind speed increasing. At the set point of wind speed, the generating power reaches the rated power of the turbine. If the wind speed continues to rise, the generator output power remains constant at the design limit. Due to safety consideration, the turbine is shut down at speeds exceeding cut-out wind speed.

1.2 Blade Aerodynamics

The wind velocity conditions at a blade crosssection are illustrated in Fig. 3.4. The velocity 
components, include the wind speed, the flow factors and rotational speed of the rotor, will determine the angle of attack. Using angle of attack, the forces on a blade element is calculated by means of two-dimensional aero foil characteristics. Then the blade element momentum (BEM) method can be used to predict the blade forces produced due to the interaction of blades with the wind. The inflow wind velocity perpendicular to the rotor plane is $\mathrm{Vw}$. When the wind passes through the rotor plane, this wind speed becomes reduced by an amount $\mathrm{aVw}$ due to axial interference. The blade rotates at angular velocity $\omega$. Thus, a blade element at

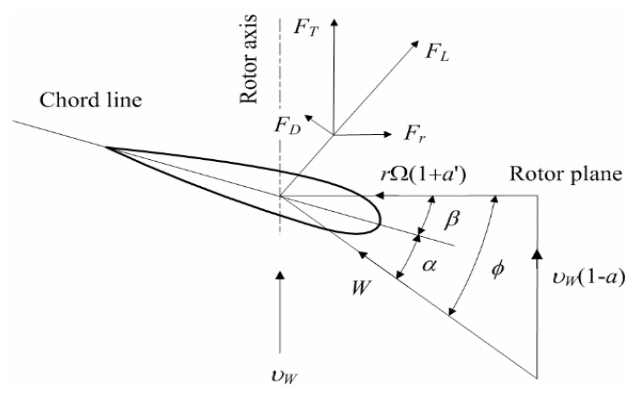

Fig.3.4. Blade element velocities and force at a blade dross-

a distance $r$ from the rotor axis will be moving at a speed $\omega_{r}$ in the rotor plane. When the wind passes

through the rotor plane and interacts with the moving rotor, a tangential velocity of the wake a' $\Omega r$ is introduced. Then the net tangential flow velocity experienced by the blade element is $\left(1+a^{\prime}\right)$ wr. The

resultant relative velocity at the blade is

$\left.W=\sqrt{V_{w}^{2}\left(1-a^{2}\right)+\omega^{2} r^{2}\left(1+a^{2}\right)^{2}} \quad 1.4\right)$

Where $\mathrm{W}$ is the resultant relative velocity at the blade, $a$ and $a$ ' is the flow factors and $r$ is the distance of blade element from the rotor axis. The resultant relative velocity gives rise to aerodynamic forces on the blade, therefore a lift force is $\mathrm{F}_{\mathrm{L}}=0.5 \rho \mathrm{cW}^{2} \mathrm{C}_{\mathrm{L}}$

And drag force is $\mathrm{F}_{\mathrm{D}}=0.5 \rho \mathrm{cW} \mathrm{W}^{2} \mathrm{C}_{\mathrm{D}}$

Where CL is lift coefficient, CD is drag coefficient and $\mathrm{c}$ is the chord length of the blade.

In the pitch-adjusting variable-speed wind turbines, the angle of attack, $\alpha_{v}$ decreases when the pitch

angle, $\beta$ increases, as shown in Fig. 3.5. The lift force, $\mathrm{F}$ decreases as well and this causes reduction of the mechanical power of the wind turbine.

According to the pitch angle control, the initial angle below rated wind speed is the optimized value, $\beta$ opt. The optimized pitch angle can be defined using the BEM method from the incoming wind and it is in very few degrees around zero. As shown in Fig. 3.3, the power of the wind turbines will increase at the increasing wind. The wind turbine must be protected against mechanical overloads and possible risk of damages at strong wind. This is achieved by pitching the blades into the position where a part of incoming wind will pass by the wind turbine. The power will be kept at its rated value at this region. By pitching, the thrust is reduced. Fig. 3.4. shows the curve of the pitch angle versus incoming wind speed which is computed with use of the BEM method for the given 1.5 MW wind turbine [16].

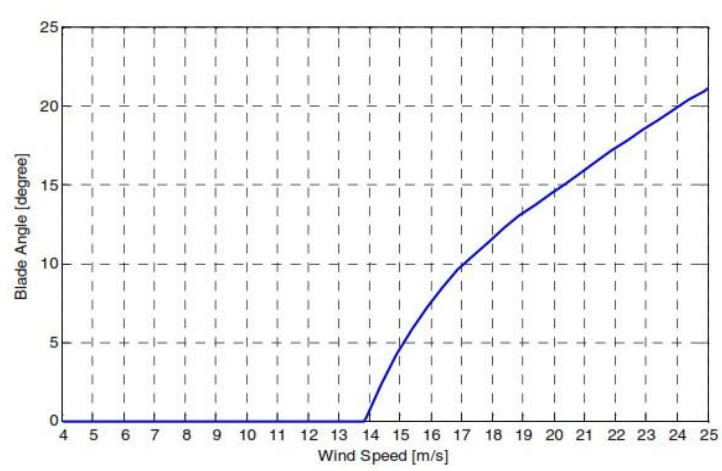

Fig.3.5. Generic blade pitch angle 


\section{2-Control}

The main control objective is to perform an optimal wind power capture while avoiding strong loads on the drive train shafts. As conventional pitch control usually use PI controller, the mathematical model of the system should be known well. A fuzzy logic pitch angle controller is developed in this paper, in which it does not need well known about the system and the mean wind speed is used to compensate the non-linear sensitivity. The fuzzy logic control strategy may have the potential when the system contains strong non-linearity, such as wind turbulence is strong, or the control objectives include fatigue loads.

Conventionally, proportional integral (PI) pitch angle controller is used to maintain the output power of the wind turbine at its rated value by adjusting the pitch angle of the blades, which provides an effective means of regulations in strong wind speeds. Fig. 1 shows a conventional simplified PI

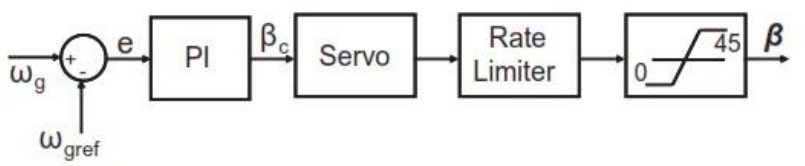

Fig. 0.1 Control scheme of the conventional PI pitch angle controller

pitch angle controller, which regulates the output in accordance with the error (e) between generator rotor speed (u) and its upper limit (reference) value. The gains of the controller $(\mathrm{KgP}$ and $\mathrm{K})$ are optimized for the optimum power generation from the wind power generation system during above rated wind incidents using trial and error method. KP and KII are obtained as 500 and 10, respectively. The upper limit value is chosen in such a way that a minimum possible reference value enables generation of maximum possible power (1 p.u.). The minimum value is expected to be chose as higher generator rotor speed is vulnerable to power system stability. For the specific wind turbine system adopted in [9], this reference value has been chosen as 1.21 p.u. The error signal is sent to the PI pitch angle controller, which produces the command pitch angle $\left(\beta_{\mathrm{c}}\right)$. It reduces the turbine operating efficiency to minimize power coefficient so that the generator maintains its control speed value.

Pitch servos are employed for proper positioning of the blades, which is modelled by using a first order delay system. The pitch actuation system cannot respond instantly. That is the reason why servo delay of $0.25 \mathrm{~s}$ has been chosen. The pitch actuation system is also limited by its actuation speed. A rate limiter for pitch rate of change of $\pm 3 \%$ has also been added to get more realistic responses from the

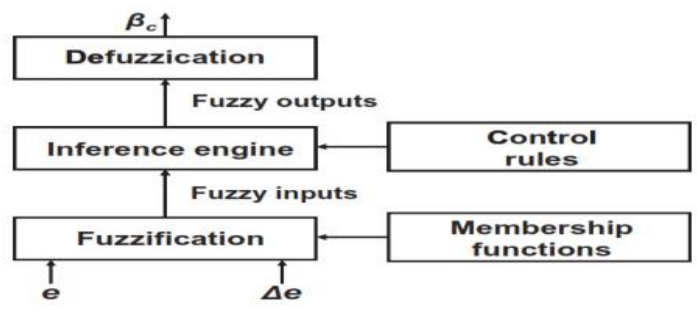

Fig. 0.3 Block Diagram of an FLS. pitch angle control system [8]. It gives the value of pitch angle (b).

\subsection{Fuzzy logic pitch angle controller}

Wind generators may operate above rated wind speed or below rated wind speed (the rated wind speed in this study is $13.3 \mathrm{~m} / \mathrm{s}$ ). Two fuzzy logic systems (FLSs) have been incorporated in the pitch angle controller for the operation of wind turbine above the rated wind incident (FLS-A) and below the rated wind incident (FLS-B). In this paper, a fuzzy logic pitch angle controller is proposed on the motivation of better smoothing performance with a lesser drop in output power. Pitch angle controller with the FLS is advantageous in a numerous ways. Wind turbine system is highly non-linear with many uncertain factors like meteorological conditions and continuously varying ac system loads [10]. It also contains some unknown ambiguous dynamics which makes accurate dynamic modeling of a wind turbine system difficult or even impossible [11]. However, the rules of the FLS possess expert adaptability and learning capability to reason precisely with imprecise, uncertain, incomplete and non-linear data from a wind turbine system [12]. Moreover, It is cheap, reliable, robust and energy efficient. An FLS comprises of three basic blocks, namely, Fuzzification, Inference system and Defuzzification as shown in Fig. 2 [13]. An FLS cannot handle crisp input signals, rather needs to describe them in fuzzy terms. Therefore, the crisp input signals have to be expressed in terms of membership function of the fuzzy sets, and then the input variables are processed to determine the degree which the input variables belong to, known as Fuzzification.

Membership functions are applied as a means of controller tuning and range between 0 and 1 . Membership functions are chosen in such a way that these reflect the characteristics of the input variables and meet the requirements of the controller. The fuzzy inference includes the process of fuzzy logic operation, fuzzy rule implication and aggregation. In the fuzzy inference system, the fuzzified input variables are processed with 'AND' fuzzy operators (selecting minimum of the input membership functions) and the IF-THEN rule implementation, which are based on expert knowledge of the control problem to be dealt with. These rules are, in fact, the control strategy of the system and describe the actions that are required for all conceivable combinations of memberships.

Fuzzy sets representing the outputs of each rule are then combined into a single fuzzy set, known as aggregation. The maximum aggregate method, taking the maximum value of all the output fuzzy sets to form a single fuzzy set, has been used in this paper. The desired output signal of the FLS is then

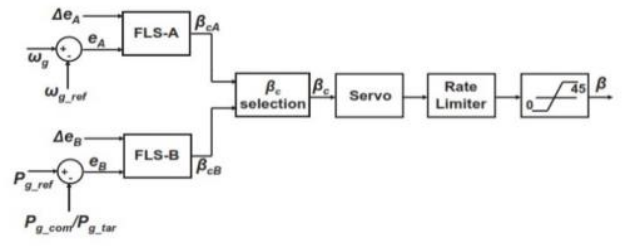

Fig. 0.2 Control scheme of the proposed fuzzy logic 
transformed into a crisp value. But at any time several rules will fire, each of them makes a suggestion as to how the output signal should be changed. Hence the defuzzification combines the results of all the rules and finds a crisp value. The Centre of Gravity (CoG) method is used for defuzzification, which returns the centre of the area under the curve representing the aggregated output fuzzy set. Fig. 3 shows the proposed controller with two combined FLS: FLS-A and FLS-B. Design strategies of FLS-A and FLS-B are described below. 1.3.1 FLS-A

When the wind turbine operates during the wind incident above the rated value, the generator rotor speed exceeds the control speed value. In this mode of operation, the controller has got nothing to do

a

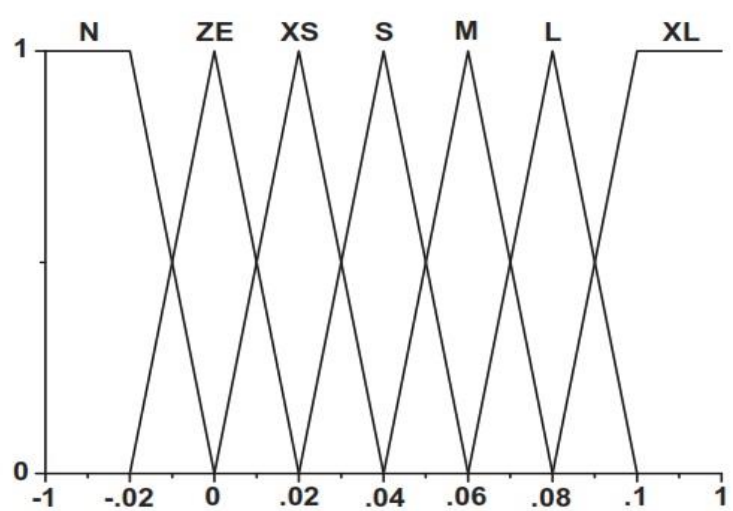

b

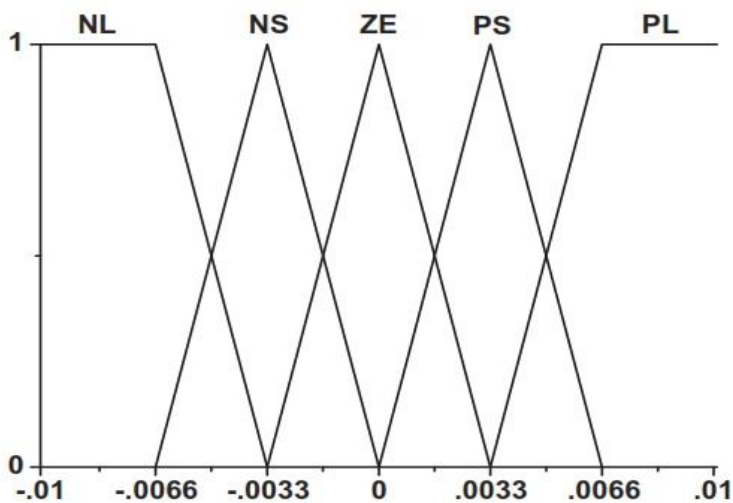

c

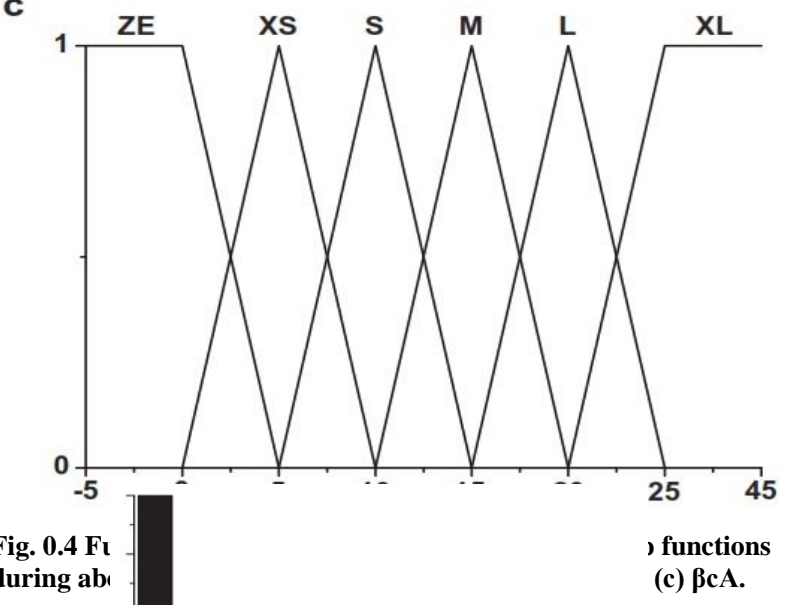
during ab

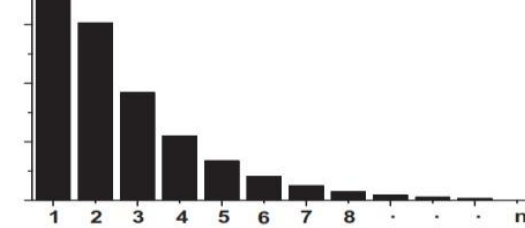

regarding power fluctuation minimization, because generator produces currents at its maximum rating irrespective of wind speed fluctuation, which ensures generation of constant wind power. The only concern of the controller is to shed mechanical power for preventing the generator rotor from going above the control speed limit value 1.21 p.u. The FLS-A has been proposed to incorporate the command pitch angle controller (b), which is only active in this mode of operation.), generator rotor speed variation from its reference value (eTo obtain command pitch angle from the FLS A (bA cA) and its variation during a sampled time (De) are used as inputs. As control action of the FLS-A would be shedding mechanical power to limit the A generator rotor speed to a control speed value, using generator rotor speed as the control input of the FLS-A is a Table 1

Rules of FLS-A.

\begin{tabular}{lllllll}
\hline$\beta_{C A}$ & & $\Delta e_{A}$ & & & & \\
\cline { 3 - 7 } & & NL & NS & ZE & PS & PL \\
\hline$e_{A}$ & NR & ZE & ZE & ZE & ZE & ZE \\
& ZE & ZE & ZE & ZE & ZE & ZE \\
& $\mathrm{XS}$ & ZE & XS & XS & XS & S \\
& $\mathrm{S}$ & $\mathrm{XS}$ & $\mathrm{S}$ & $\mathrm{S}$ & $\mathrm{S}$ & $\mathrm{M}$ \\
& $\mathrm{M}$ & $\mathrm{S}$ & $\mathrm{M}$ & $\mathrm{M}$ & $\mathrm{M}$ & $\mathrm{L}$ \\
& $\mathrm{L}$ & $\mathrm{M}$ & $\mathrm{L}$ & $\mathrm{L}$ & $\mathrm{L}$ & $\mathrm{XL}$ \\
& $\mathrm{XL}$ & $\mathrm{L}$ & $\mathrm{XL}$ & $\mathrm{XL}$ & $\mathrm{XL}$ & $\mathrm{XL}$ \\
\hline
\end{tabular}

means of direct control method. It gives more flexible control over the system by enabling constant observation on generator rotor speed.

In this paper, triangular membership functions with overlapping are used, as they are easy to implement, quicker to process and gives more sensitivity especially as variables approach to zero. Overlapping causes firing of more than one rule, which is a key feature of fuzzy systems. Fig. 4 shows the input and output membership functions. The linguistic variables are represented by $\mathrm{N}$ (Negative), ZE (Zero), XS (Extra Small), S (Small), M (Medium), L (Large), XL (Extra Large), NS (Negative Small), NL (Negative Large), PS (Positive Small) and PL (Positive Large). The FLSA has 35 rules that are built by crossing the fuzzy sets. The same weight has been considered for all the rules. The rules are sorted into groups depending on the signals they deal with, which are listed in Table 1 . The ith fuzzy rule is expressed by [14] as Rule $i$ if if $e_{A}(n)$ is $A_{a}$ and $\Delta e_{A}(n)$ is $B_{b}$. Then $\beta_{\mathrm{cA}}(\mathrm{n})$ is $\mathrm{C}_{\mathrm{c}}$

$$
a=1,2 \ldots 7 b=1,2 \ldots 5 c=1,2 \ldots 350.1)
$$

Where $\mathrm{A}_{\mathrm{a}}$ and $\mathrm{B}_{\mathrm{b}}$ denote the antecedents and $\mathrm{C}_{\mathrm{c}}$ are the consequent part.

Command pitch angle $\left(\beta_{\mathrm{cA}}\right)$ is calculated by applying CoG method as [14] $\left.\beta_{e A}(n)=\sum_{i=1}^{25} \omega_{i} C_{C} / \sum_{i=1}^{25} \omega_{i} 0.2\right)$

Where $\omega_{i}$ denotes the grade for the antecedent,

which is the product of grade for the antecedents of each rule. 


\subsubsection{FLS-B}

On the other hand, when the wind turbine operates during the wind incident below the rated value, there is no generation of pitch angle. Any variation in wind speed can cause high fluctuations in wind power. To smooth the fluctuating wind power, two methods have been proposed to incorporate the FLS-B controller by generating command pitch angle (bcB).

\subsubsection{Method 1}

The smoothing technique of method 1 is to determine) from the reference output power (the output power from the wind turbine with the conventional PI controller has been considered as reference output power ( $\mathrm{P}$ a command output power (Pg_com)) and generate pitch angle in such a way so that the generated output power can follow the

a
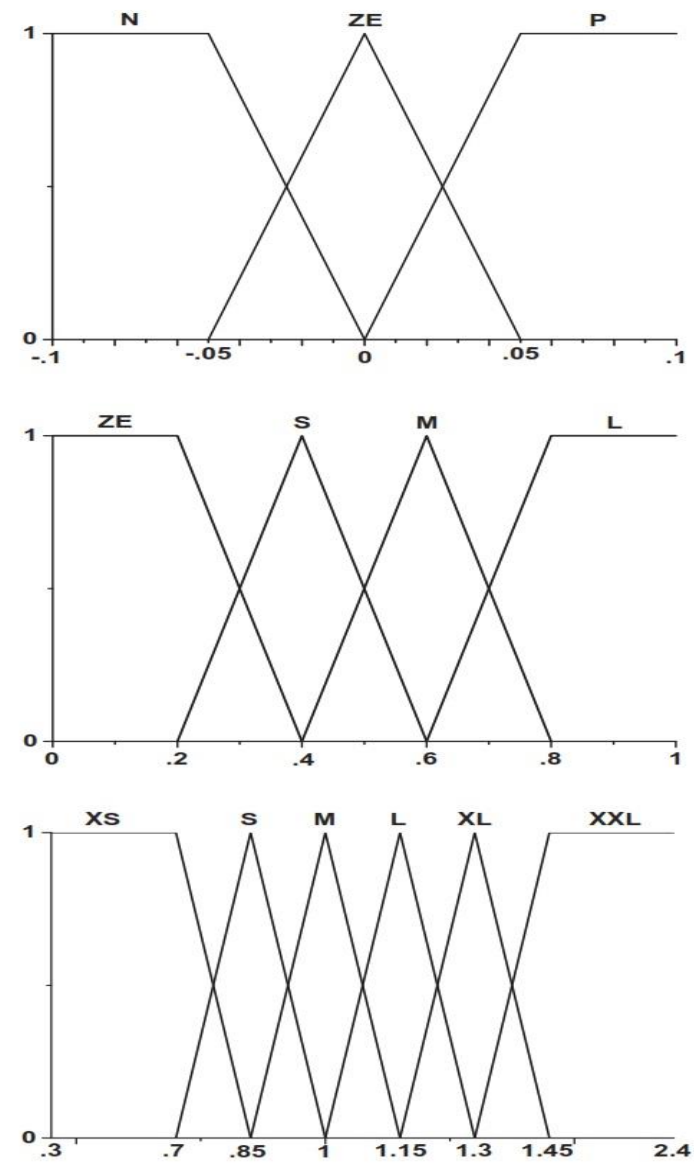

Fig. 0.6 Fuzzy sets and their corresponding membership functions for determining a proper correction factor: (a) eB1, (b) Pg-ref and (c) k.

command output power. To accomplish this,

- Command output power must be smooth.

- Smoothing is achieved by the generation of pitch angle with some drop in output power. As the generated output power is always lower than the reference output power, command output power must be ensured to be lower than the reference value so that the fuzzy rules for the generation of pitch angle are effectively set up by ensuring control input of variation of reference output power from the command output power in FLS-B falls in positive domain.

Table 2

\begin{tabular}{lllll}
\multicolumn{2}{l}{ Rules for determining correction factor. } \\
\hline$k$ & & $e_{B 1}$ & & \\
\cline { 2 - 5 } & $\mathrm{N}$ & $\mathrm{ZE}$ & $\mathrm{P}$ \\
\hline$P_{\text {g_ref }}$ & $\mathrm{ZE}$ & $\mathrm{XS}$ & $\mathrm{S}$ & $\mathrm{XXL}$ \\
& $\mathrm{S}$ & $\mathrm{XS}$ & $\mathrm{S}$ & $\mathrm{XL}$ \\
& $\mathrm{M}$ & $\mathrm{XS}$ & $\mathrm{S}$ & $\mathrm{L}$ \\
$\mathrm{L}$ & $\mathrm{S}$ & $\mathrm{S}$ & $\mathrm{L}$ \\
\hline
\end{tabular}

As the initial step, the smoothed version of reference output power, known as 'EMA command output power $(\mathrm{P})$ ' has been generated. This command value at any instant $t$ is given as [15]

$\left.P_{g-\operatorname{comx}}=\alpha C_{t}+(1-\alpha) P_{t} 0.3\right)$

where $\mathrm{C}$ is current value and $\mathrm{P}$ is previous period's value of the reference output power and $\mathrm{a}$ is the smoothing constant.

In this paper, 12 periods of average value (each of 1 s) is used in simulation. So, the EMA starts from 12 $\mathrm{s}$ when 12 period's data are available. Smoothing constant (a) is chosen as 0.8 , which indicates that $80 \%$ weight has been considered for the data of present period in comparison with $20 \%$ weight to the previous period's data. The trend of weight assigned to the previous period's data can be realized by expanding Eq. (3)

$P_{g-\omega 0 n_{1}^{p}}=\alpha C_{t}+(1-\alpha)\left[\alpha C_{t-1}+(1-\alpha) P_{t-1}\right]=$ $\omega C_{t}+\alpha(1-\alpha) C_{t-1}+(1-\alpha)^{2} P_{t-1}=\alpha \sum_{i=0}^{t-1}(1-$ $\alpha)^{i} C_{t-1}+(1-\alpha)^{t} P_{t-1}$

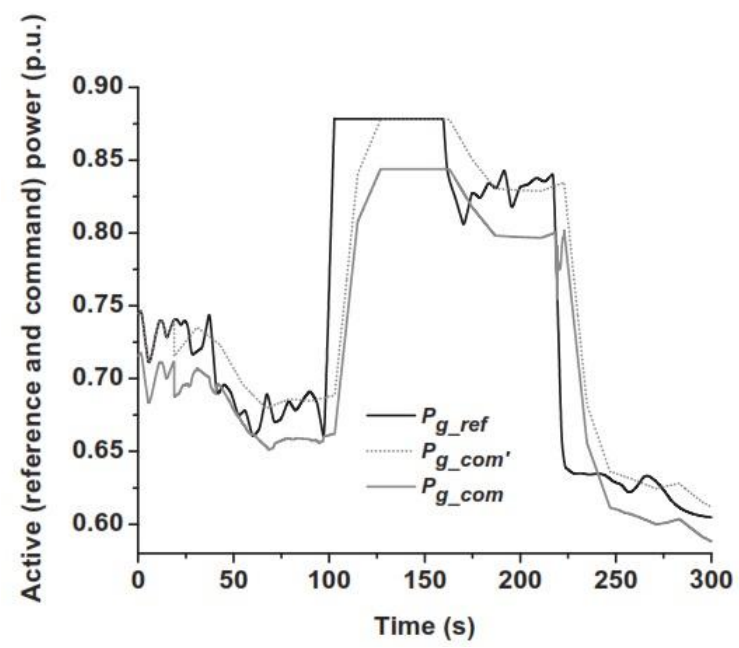

Fig. 0.7 Obtaining command output power from EMA command output power by the generation of correction factor (k).

The term $(1-\alpha)^{\mathrm{I}}$ indicates that weights of the previous period's data are exponentially decreasing. The distribution of the EMA weights for $n$ samples is shown in Fig. 5.

The superiority of the EMA over other smoothing techniques is that the EMA can follow wind speed more rapidly because it uses its data of previous periods for next calculation [8]. 
To ensure the command output power (Pg_com) to be lower value than the reference value, a concept of correction factor $(\mathrm{k})$ has been introduced, which is related to the command output power (Pg_com) in the following manner.

$P_{g-\operatorname{com}}=k \times P_{g-\operatorname{com}} \quad 0.5$ )

The correction factor has bee $\mathrm{n}$ assessed by applying fuzzy reasoning. Variation of reference output power from the command output power $\left(\mathrm{e}_{\mathrm{B} 1}\right)$ and reference output power (Pg_ref) are used as inputs. The system has triangular membership functions with overlapping. Fig. 6 shows the input and output membership functions. The linguistic variables are represented by N, ZE, P (Positive), S, M, L, XS, XL and XXL (Double Extra Large).

The system has got 12 rules, which are formed in

a

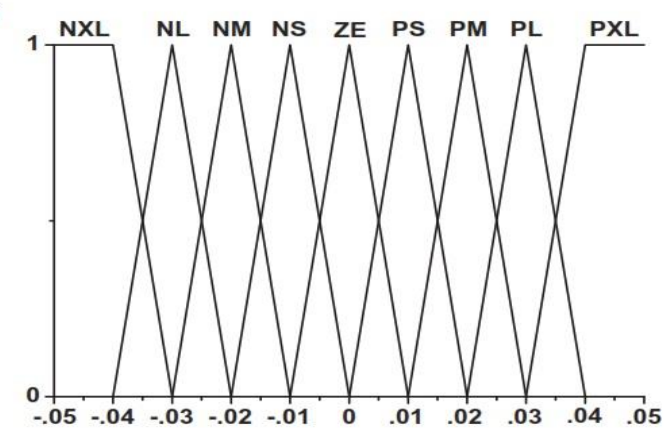

b

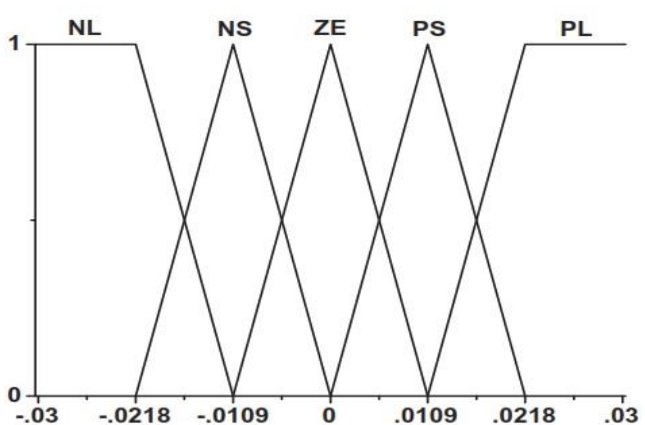

c

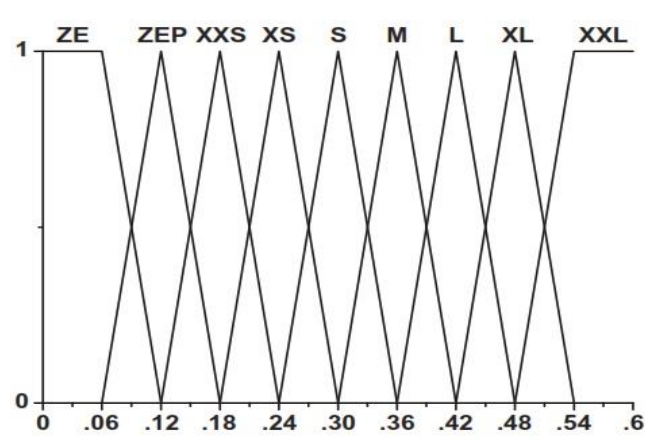

Fig. 0.8 Fuzzy sets and their corresponding membership functions during below rated wind incidents using Method1: (a) eB1, (b) $\triangle \mathrm{eB1}$ and (c) $\beta \mathrm{cB} 1$.

similar way of Eq. (1) and listed in Table 2. Correction factor $(\mathrm{k})$ has been crisped by the $\mathrm{CoG}$ defuzzification method using Eq. (2).

Fig. 7 shows that the command output power (P g_com) achieved is lower than the reference output power (Pg_ref) over the whole $300 \mathrm{~s}$ period except for the period during the rapid change in operating points from the EMA command output power $(\mathrm{P})$ by the generation of correction factor $(\mathrm{k})$, where the role of $\mathrm{k}$ is non-significant because output power simply switches to a different operating point and needs not to be smoothed. The role of $\mathrm{k}$ is not accountable during above rated wind incidents as well, because output power is generated at its maximum rating and needs not to be smoothed either.

To obtain command pitch angle from FLS-B using Method 1 (b), variation of reference output power from the command output power (ecB1B1) and its variation during a sampled time (De) are used as inputs. The system has triangular membership functions with overlapping. Fig. 8 shows the input and output membership functions. The linguistic variables are represented by NXL (Negative Extra Large), NL (Negative Large), NM (Negative Medium), NS (Negative Small), ZE, PS, PM (Positive Medium), PL, PXL (Positive Extra Large), ZEP (Zero Plus), XXS (Double Extra Small), XS, S, $\mathrm{M}, \mathrm{L}, \mathrm{XL}$ and XXL.

FLS-B has 45 rules, which are formed in similar way of Eq. (1) and listed in Table 3. Command pitch angle (b) has been crisped by the $\mathrm{CoG}$ defuzzification method using Eq. (2).

\subsubsection{Method 2}

Another method is proposed to minimize wind power fluctuations during below rated wind incidents for the FLS-B. The following steps have been carried out as a means of design strategy:

- Generated wind power (P) has been categorized into the stages with the steps of 0.05 p.u., which is defined as 'Power Stage (PS)' as shown in Table 4. The lower limit value of each corresponding PS is taken as a target value $(\mathrm{P})$ for the controller.

- If the generated wind power falls under a PS, a

Table 4

Rules of FLS-B (Method 2).

\begin{tabular}{lllllll}
\hline$\beta_{C B 2}$ & & $\Delta e_{B 2}$ & & & & \\
\cline { 3 - 6 } & & $\mathrm{NL}$ & $\mathrm{NS}$ & $\mathrm{ZE}$ & $\mathrm{PS}$ & $\mathrm{PL}$ \\
\hline$e_{\text {B2 }}$ & $\mathrm{ZE}$ & $\mathrm{XXS}$ & $\mathrm{S}$ & $\mathrm{ZE}$ & $\mathrm{S}$ & $\mathrm{L}$ \\
& $\mathrm{ZEP}$ & $\mathrm{XS}$ & $\mathrm{M}$ & $\mathrm{ZEP}$ & $\mathrm{M}$ & $\mathrm{XL}$ \\
& $\mathrm{XXS}$ & $\mathrm{S}$ & $\mathrm{L}$ & $\mathrm{XXS}$ & $\mathrm{L}$ & $\mathrm{XXL}$ \\
& $\mathrm{XS}$ & $\mathrm{M}$ & $\mathrm{XL}$ & $\mathrm{S}$ & $\mathrm{XL}$ & $\mathrm{XXL}$ \\
& $\mathrm{S}$ & $\mathrm{L}$ & $\mathrm{XXL}$ & $\mathrm{S}$ & $\mathrm{XXL}$ & $\mathrm{XXL}$ \\
$\mathrm{M}$ & $\mathrm{XL}$ & $\mathrm{XXL}$ & $\mathrm{L}$ & $\mathrm{XXL}$ & $\mathrm{XXL}$ \\
& $\mathrm{L}$ & $\mathrm{XXL}$ & $\mathrm{XXL}$ & $\mathrm{L}$ & $\mathrm{XXL}$ & $\mathrm{XXL}$ \\
& $\mathrm{SL}$ & $\mathrm{XXL}$ & $\mathrm{XXL}$ & $\mathrm{XXL}$ & $\mathrm{XXL}$ & $\mathrm{XXL}$ \\
& $\mathrm{XXL}$ & $\mathrm{XXL}$ & $\mathrm{XXL}$ & $\mathrm{XXL}$ & $\mathrm{XXL}$ & $\mathrm{XXL}$ \\
\hline
\end{tabular}

Table 3

\begin{tabular}{lllllll}
\hline \multirow{3}{*}{$\beta_{C B 1}$} & & $\Delta e_{B 1}$ & & & & \\
\cline { 3 - 6 } & & $\mathrm{NL}$ & $\mathrm{NS}$ & $\mathrm{ZE}$ & $\mathrm{PS}$ & $\mathrm{PL}$ \\
\hline$e_{B 1}$ & $\mathrm{NXL}$ & $\mathrm{XXS}$ & $\mathrm{S}$ & $\mathrm{ZE}$ & $\mathrm{S}$ & $\mathrm{L}$ \\
& $\mathrm{NL}$ & $\mathrm{XS}$ & $\mathrm{M}$ & $\mathrm{ZEP}$ & $\mathrm{M}$ & $\mathrm{XL}$ \\
& $\mathrm{NM}$ & $\mathrm{S}$ & $\mathrm{L}$ & $\mathrm{XXS}$ & $\mathrm{L}$ & $\mathrm{XXL}$ \\
& $\mathrm{NS}$ & $\mathrm{M}$ & $\mathrm{XL}$ & $\mathrm{S}$ & $\mathrm{XL}$ & $\mathrm{XXL}$ \\
& $\mathrm{ZE}$ & $\mathrm{L}$ & $\mathrm{XXL}$ & $\mathrm{S}$ & $\mathrm{XXL}$ & $\mathrm{XXL}$ \\
& $\mathrm{PS}$ & $\mathrm{XL}$ & $\mathrm{XXL}$ & $\mathrm{L}$ & $\mathrm{XXL}$ & $\mathrm{XXL}$ \\
& $\mathrm{PM}$ & $\mathrm{XXL}$ & $\mathrm{XXL}$ & $\mathrm{L}$ & $\mathrm{XXL}$ & $\mathrm{XXL}$ \\
& $\mathrm{PL}$ & $\mathrm{XXL}$ & $\mathrm{XXL}$ & $\mathrm{XXL}$ & $\mathrm{XXL}$ & $\mathrm{XXL}$ \\
& $\mathrm{PXL}$ & $\mathrm{XXL}$ & $\mathrm{XXL}$ & $\mathrm{XXL}$ & $\mathrm{XXL}$ & $\mathrm{XXL}$ \\
\hline
\end{tabular}


pitch angle would be actuated to shed mechanical power and limit the wind power to Pg_tar. For example, if $\mathrm{Pg}=0.78$ p.u. at any instant, a pitch angle is actuated to make $\mathrm{Pg}=0.75$ p.u. (see PS3in Table 3).

To obtain command pitch angle from FLS-B using Method $2\left(\beta_{\mathrm{cB} 2}\right)$, variation of reference output power from the target value selected by FLS-B $\left(\mathrm{e}_{\mathrm{B} 2}\right)$ and its variation during a sampled time $\left(\Delta \mathrm{e}_{\mathrm{B} 2}\right)$ are used as inputs. Control action of FLS-B would be limiting output power to a certain target value instead of generator rotor speeds, which make the control design easier because the use of the rotor speed would cause non-linear.

This system also has triangular membership functions with overlapping. Fig. 9 shows the input and output membership functions. The linguistic variables are represented by ZE, ZEP, XXS, XS, S, M, L, XL, XXL, NS, NL, PS and PL.

Table 5

\begin{tabular}{lll} 
Power stages. & & \\
\hline Power stage & Range & $P_{\text {g_tar }}$ \\
\hline PS1 & $0.9<\mathrm{Pg} \leq 0.85$ & 0.85 \\
PS2 & $0.85<\mathrm{Pg} \leq 0.8$ & 0.8 \\
PS3 & $0.8<\mathrm{Pg} \leq 0.75$ & 0.75 \\
PS16 & $0.15<\mathrm{Pg} \leq 0.1$ & 0.1 \\
PS17 & $0.1<\mathrm{Pg} \leq 0.05$ & 0.05 \\
PS18 & $0.05<\mathrm{Pg} \leq 0$ & 0 \\
\hline
\end{tabular}

The range of the command pitch angle output is different for each corresponding PS. It generally requires more pitch angle generation as wind power falls in the PS of lower levels. The values of $\mathrm{x} 1$ and $\mathrm{x} 2$ of Fig. 9c are listed in Table 5. The same values

\section{Table 6}

Command pitch angle range for FLC-B (Method 2).

\begin{tabular}{llllll}
\hline Power stage & $x 1$ & $x 2$ & Power stage & $x 1$ & $x 2$ \\
\hline PS1 & 0 & 0 & PS12 & 0.5 & 1.4 \\
PS2 & 0.2 & 0.6 & PS13 & 0.5 & 1.6 \\
PS3 & 0.3 & 0.7 & PS14 & 0.5 & 1.8 \\
PS4-PS7 & 0.3 & 0.9 & PS15 & 0.6 & 2 \\
PS8-PS9 & 0.4 & 1.1 & PS16 & 0.8 & 6 \\
PS10 & 0.5 & 1.2 & PS17 & 1.5 & 15 \\
PS11 & 0.5 & 1.3 & PS18 & 45 & 45 \\
\hline
\end{tabular}

of $\mathrm{x} 1$ and $\mathrm{x} 2$ for PS1 and PS18 refer to singleton output membership function.

FLS-B has 45 rules, which are formed in similar way of Eq. (1) and listed in Table 6. Command pitch angle (b) is crisped by the $\mathrm{CoG}$ defuzzification method using Eq. (2).

\section{Conclusions}

Wind energy has the potential to play an important role in the future energy supply in many areas of the world. Within the last 10 years, wind turbine technology has reached a very reliable and sophisticated level. The growing international market will lead to further improvements, such as large wind turbines or new system applications, e.g. offshore wind farms. These improvements will lead to further cost reductions, and for the medium term, wind energy will be able to compete with conventional fossil fuel power generation technology. Further research, however, will be required in many areas, for example, regarding the network integration of a high penetration of wind energy.

Pitching the individual blades (IPC) of a horizontalaxis wind turbine allows control of asymmetric aerodynamic loads, which in turn influences structural loads in the nonrotating frame such as tower side-side bending. These loads are not easily controlled by traditional collective pitch control (CPC).

Due to the complexity of the wind energy technology, however, this paper mainly aims at presenting a brief overview of the relevant wind turbine and an example of IPC by using Fuzzy logic as one of the pitching control methods.

\section{References}

1. Caselitz P, Kleinkauf W, Kruger T, Petschenka J, Reichardt M, Storzel K(1997) . Reduction of fatigue loads on wind energy converters by advanced control methods. Proceedings of the European Wind Energy Conference, Dublin,; 555-558.

2. Bossanyi EA.( 2002) Individual Blade Pitch Control for Load Reduction Wind. John Wiley \& Sons, Ltd.

3. M.A. Chowdhury, N. Hosseinzadeh, W.X. (2011) Shen Smoothing wind power fluctuations by fuzzy logic pitch angle controller. Elsevier Ltd.

4. Jianzhong Zhang, Ming Cheng, Zhe Chen, Xiaofan Fu, Pitch Angle Control for Variable Speed Wind Turbines. DRPT2008 6-9 April 2008 Nanjing China

5. T. Burton, D. Sharpe, N. Jenkins, E. Bossanyi, ( 2001) Wind energy handbook, John Wiley \& Sons Ltd, Chichester, UK,

6. DNV/Ris $\varnothing,(2002)$. Guidelines for design of wind turbines, 2nd ed., Jydsk Centraltrykkeri, Denmark,

7. S. Suryanarayanan, A. Dixit, Control of large wind turbines: Review and suggested approach to multivariable design, Proc. of the American Control Conference 2005, Portland, USA, pp. 686-690.

8. Kamel RA, Chaouachi A, Nagasaka K. Wind power smoothing using fuzzy logic pitch controller and energy capacitor system for improvement Micro-Grid performance in islanding mode. Energy 2010;35(5):2119-29.

9. SimPowerSystems e; model and simulate electrical power systems. User's guide. Natick (MA): The Mathworks Inc; 2010.

10. Chen Z, Gomez SA, McCormick M. A fuzzy (2000) logic controlled power electronic system for variable speed wind energy conversion systems. Int Conf Paw Elect Speed Drives;

11. Galdi V, Piccolo A, Siano P. Designing an adaptive fuzzy controller for maximum wind energy extraction. IEEE Trans Ener Conv 2008;23(2):559e69.

12. Simoes MG, Bose BK, Spiegel RJ. Design and performance evaluation of a fuzzy-logic-based variable-speed wind generation system. IEEE Trans Ind Appl 2008;33(4):956e65.

13. Krichen L, Francois B, Ouali A. A fuzzy logic supervisor for active and reactive power control of a 
fixed speed wind energy conversion system. Elect Pow Sys Res 2008; 78(3):418e24.

14. Senjyu T, Sakamoto R, Urasaki N, Funabashi $\mathrm{T}$, Fujita H, Sekine H. Output power leveling of wind turbine generator for all operating regions by pitch angle control. IEEE Trans Ener Conv 2006;21(2):467e75.

15. NIST/SEMATECH e e-Handbook of Statistical Methods. Available at (2-12-2015). from:

16. http://www.itl.nist.gov/div898/handbook/index.htm;

17. Jianzhong Zhang, Ming Cheng, Pitch Angle Control for Variable Speed Wind Turbines. DRPT2008 6-9 April 2008 Nanjing China
18. B. Boukhezzar, H. Siguerdidjane, Comparison between linear and nonlinear control strategies for variable speed wind turbines, www.elsevier.com/locate/conengprac, Control Engineering Practice 18 (2010) 1357-1368

19. Thomas Ackermann, Lennar SoÈder, Wind energy technology and current status: a Review, Renewable and Sustainable Energy Reviews 4 (2000) 315 374 ”. 